\title{
Correção do astigmatismo irregular com lente intraocular tórica em um paciente com catarata e degeneração marginal pelúcida: relato de caso
}

\author{
Toric intraocular lens implantation for cataract and \\ irregular astigmatism related to pellucid \\ marginal degeneration: case report
}

Ana Luiza Biancardi ${ }^{1}$, Aileen Walsh${ }^{1}$, Rodrigo de Pinho Paes Barreto ${ }^{1}$, Armando Stefano Crema ${ }^{1,3}$

\section{Resumo}

A degeneração marginal pelúcida (DMP) é uma rara ectasia corneana cuja progressão resulta em astigmatismo irregular e baixa visual não corrigidos com óculos ou lentes de contato. O presente relato descreve um paciente com catarata e DMP que foi tratado com facoemulsificação e implante de lente intraocular tórica com recuperação da acuidade visual em ambos os olhos.

Descritores: Doenças da córnea; Astigmatismo/cirurgia; Implante de lente intraocular; Extração de catarata; Relatos de casos

\begin{abstract}
Pellucid marginal degeneration $(P M D)$ is a rare corneal ectasia and its progression leads to irregular astigmatism and low vision that can not have spectacles or contact lens correction. This report describes a patient with low vision due to cataract and PMD that was treated with phacoemulsification and implantation of a toric intraocular lens with a satisfactory visual acuity outcome.
\end{abstract}

Keywords: Corneal diseases; Astigmatism/surgery; Lens implantation, intraocular; Cataract extraction; Case reports

\footnotetext{
${ }^{1}$ Walsh \& Crema Clínica e Microcirurgia Ocular - Rio de Janeiro (RJ), Brasil;

${ }^{2}$ Hospital dos Servidores do Estado do Rio de Janeiro (HSE) - Rio de Janeiro (RJ), Brasil;

${ }^{3}$ Professor Assistente da Universidade Gama Filho (UGF) - Rio de Janeiro (RJ), Brasil.

Instituição: Walsh \& Crema Clínica e Microcirurgia Ocular - Rio de Janeiro (RJ), Brasil.
}

Os autores declaram não haver conflitos de interesse

Recebido para publicação em: 12/5/2011 - Aceito para publicação em: 15/9/2011

Rev Bras Oftalmol. 2012; 71 (6): 400-2 


\section{INTRODUÇãO}

A degeneração marginal pelúcida (DMP) é uma ectasia corneana não inflamatória rara, geralmente bilateral, que afeta homens e mulheres entre 20 e $40 \operatorname{anos}^{(1)}$. A etiologia da DMP não é bem definida. A microscopia eletrônica da córnea evidenciou fibras colágenas anormalmente espaçadas e frágeis que resultam no afinamento corneano, geralmente inferior $^{(2)}$

A progressão da DMP leva à baixa visual e ao astigmatismo irregular nem sempre satisfatoriamente corrigidos com óculos ou lentes de contato. Portanto, em casos graves o tratamento cirúrgico é indicado, embora não exista consenso quanto à melhor abordagem. A eficácia e segurança da lente intraocular (LIO) tórica já são bem estabelecidas no paciente com astigmatismo regular ${ }^{(3,4)}$, mas a utilização desta LIO em casos patológicos ainda é pouco aplicada.

O presente relato descreve um paciente com baixa visual em decorrência de catarata e DMP que foi tratado com facoemulsificação e implante de LIO tórica com recuperação da acuidade visual.

\section{Relato de Caso}

Paciente masculino, 55 anos, procurou atendimento queixando-se de baixa visual e relatando "doença da córnea". O exame oftalmológico revelou acuidade visual corrigida (AVC) igual a 20/40 no olho direito (OD) e 20/70 no olho esquerdo (OE). A refração manifesta do OD era igual a - 10,00 - 4,00 X 85 e no OE era igual a - 11,00 - 3,00 X 80. A topografia corneana (OPD Scan II, Nidek) foi compatível com DMP em ambos os olhos (AO), mais avançada no OD (Figura 1). A biomicroscopia revelou catarata em $\mathrm{AO}$; a tonometria e o fundo de olho não apresentaram alterações em AO.

Foi indicada facoemulsificação com implante de LIO. O paciente realizou biometria óptica (IOL Master v.5.0, Carl Zeiss Meditec) com cálculo da LIO através da fórmula Haigis e confirmação com a fórmula Holladay II. O poder das LIOs indicadas foi
+11,50 D no OD e +13,50 D no OE. As cirurgias foram realizadas sem intercorrências com implante de LIOs hidrofóbicas tóricas de peça única (Acrysof ${ }^{\circledR}$ SN60T5, Alcon); a refração manifesta no pós-operatório foi - 1,00 - 1,00 X 105 no OD e - 0,75 X 90 no OE; no seguimento de 2 anos a acuidade visual corrigida manteve-se 20/20 com estabilidade topográfica e refracional.

\section{DisCusSÃo}

$\mathrm{O}$ astigmatismo irregular associado à ectasia corneana, em especial com alterações topográficas significativas nem sempre é corrigido com óculos ou lentes de contato.

Casos moderados e graves são tratados com cirurgias tais como ressecção em crescente, ressecção lamelar em crescente, ceratoplastia lamelar, ceratoplastia penetrante, epiceratoplastia, uso de segmentos intraestromais corneais de PMMA, incisões relaxantes limbares e implante de lentes fácicas ${ }^{(1,4-9)}$. A variedade de opções cirúrgicas demonstra a ausência de um tratamento plenamente eficaz para DMP.

Relatos prévios descreveram o uso de LIO tórica no tratamento do astigmatismo patológico, relacionado ao ceratocone ${ }^{(10)}$, $\mathrm{DMP}^{(11)}$ ou após o transplante de córnea ${ }^{(12)}$. A princípio, a LIO tórica corrigia graus leves e moderados de astigmatismo corneano até $2,06 \mathrm{D}$.

Atualmente o cirurgião é capaz de corrigir plenamente cilindros maiores desde o advento das LIOs Acrysof ${ }^{\circledR}$ Toric T6 a T9 (Alcon Inc) e das LIOs tóricas Rayner T-flex ${ }^{\circledR}$ 573T e 623T (Rayner); essas LIOs são capazes de corrigir cilindros de até $7,7 \mathrm{D}^{(13)}$. Entretanto, graus especiais têm sua indicação limitada devido ao alto custo destas LIOs.

Apesar do amplo poder de correção das LIOs tóricas, o alto astigmatismo, em especial o irregular, nem sempre é corrigido na sua totalidade e medidas adicionais podem ser tomadas para evitar o astigmatismo residual. Luck ${ }^{(11)}$ descreveu um caso de DMP e catarata tratado com uma LIO de alto poder especialmente customizada para o paciente e denominada LIO AT.Comfort 646TLC bitórica, para tratar um cilindro irregular de 10,9D.
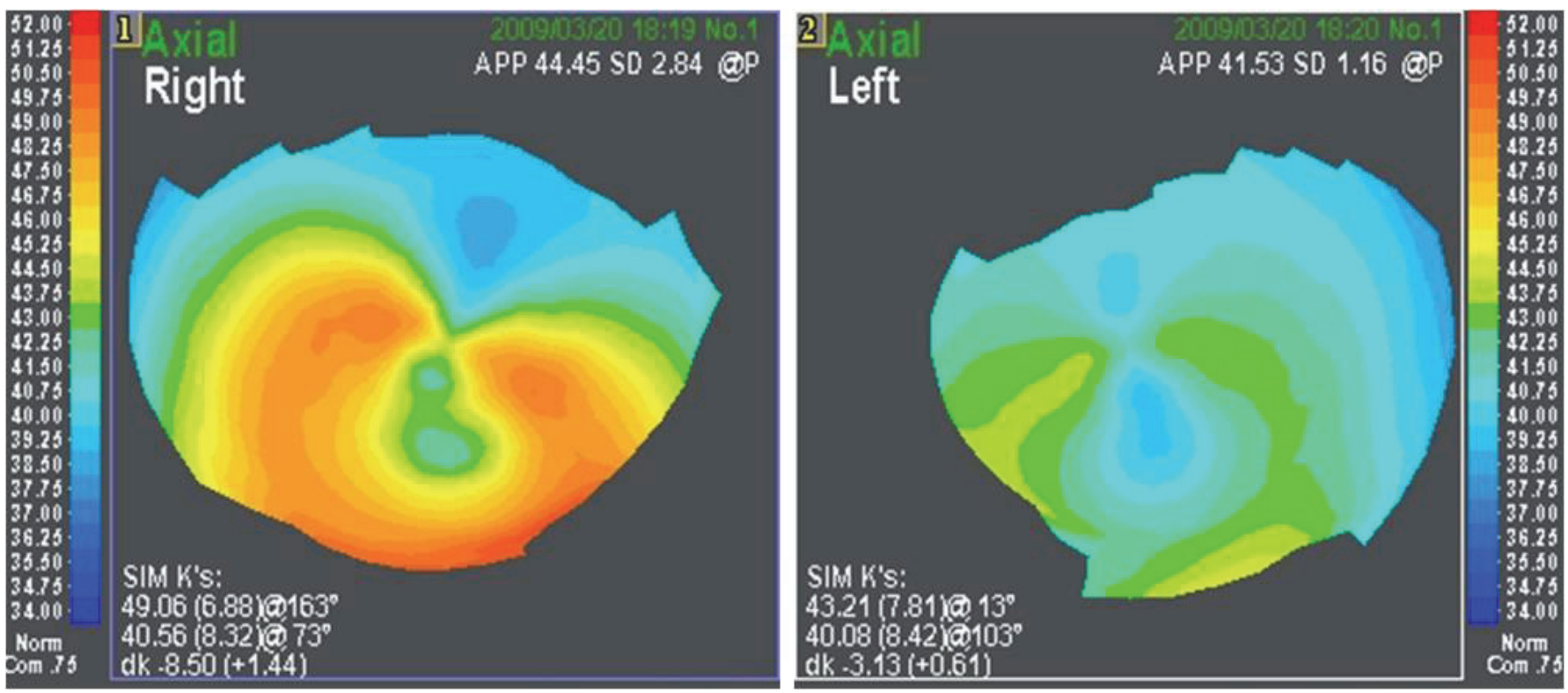

Figura 1: Degeneração marginal pelúcida; Topografia (OPD Scan II, Nidek) 
A biometria em pacientes com irregularidade da córnea pode ser difícil devido à imprecisão da medida do poder corneano e não há consenso sobre o melhor método nestes casos. Relatos prévios descreveram o uso do sistema Scheimpflug no pré-operatório de pacientes com ectasia ${ }^{(10,11)}$. No presente caso, a ceratometria nos $3 \mathrm{~mm}$ centrais na topografia e nos $2.5 \mathrm{~mm}$ centrais no IOL Master apresentou resultados reprodutíveis e não foram realizadas medidas ceratométricas por outros métodos. O poder central da córnea, mais plano, desproporcional ao comprimento axial mais longo, foi determinante para a escolha das fórmulas de quarta geração Haigis e Holladay II, que apresentaram resultados semelhantes.

No presente relato, o implante da LIO tórica foi capaz de reduzir a refração e melhorar a qualidade visual em AO com boa estabilidade do quadro após 2 anos de seguimento. A pequena miopia no OD foi intencionalmente calculada para favorecer a monovisão; o astigmatismo residual contra a regra, que não foi passível de correção com os graus disponíveis de LIOs tóricas na ocasião da cirurgia, também contribuiu para a visão de perto com resultado considerado excelente pelo paciente.

Entretanto, é importante lembrar que a ectasia corneana tende a ser uma doença progressiva com aumento gradual do astigmatismo irregular, o que pode resultar em instabilidade refracional e comprometimento da satisfação do paciente a longo prazo. Portanto, é provável que pacientes mais jovens com exames seriados demonstrando progressão da ectasia não se beneficiem deste tratamento; os candidatos à cirurgia também devem ser alertados sobre a dificuldade biométrica e possibilidade de ametropia residual.

\section{REFERÊNCIAS}

1. Mérula RV, Trindade FC. Degeneração marginal pelúcida da córnea: diagnóstico e tratamento. Arq Bras Oftalmol. 2006;69(3):447-53.

2. Rodrigues MM, Newsome DA, Krachmer JH, Eiferman RA. Pellucid marginal corneal degeneration: a clinicopathologic study of two cases. Exp Eye Res. 1981;33(3):277-88.

3. Centurion V, Lacava AC, Caballero JC. A neutralização do astigmatismo corneano durante a cirurgia da catarata por meio de lente intraocular tórica: resultados. Rev Bras Oftalmol. 2009;68(2):76-82.
4. Correia RJB, Moreira H, Netto SUL, Pantaleão GR. Performance visual após implante de LIO tórica em pacientes com astigmatismo corneano. Arq Bras Oftalmol. 2009;72(5):636-40.

5. de Vries NE, Tahzib NG, Webers CAB, Hendrikse F, Nuijts RM. Use of Verisyse/Artisan phakic intraocular lens for the reduction of myopia in a patient with pellucid marginal degeneration. Cornea. 2008;27(2):241-5.

6. Rodriguez-Prats J, Galal A, Garcia-Lledo M, De La Hoz F, Alió JL. Intracorneal rings for the correction of pellucid marginal degeneration. J Cataract Refract Surg. 2003;29(7):1421-4.

7. Millar MJ, Maloof A. Deep lamellar keratoplasty for pellucid marginal degeneration: review of management options for corneal perforation. Cornea. 2008;27(8):953-6.

8. Tzelikis PF, Cohen EJ, Rapuano CJ, Hammersmith KM, Laibson PR. Management of pellucid marginal corneal degeneration. Cornea. 2005;24(5):555-60.

9. Busin M, Kerdraon Y, Scorcia V, Zambianchi L, Matteoni S. Combined wedge resection and beveled penetrating relaxing incisions for the treatment of pellucid marginal corneal degeneration. Cornea. 2008;27(5):595-600.

10. Visser N, Gast ST, Bauer NJ, Nuijts RM. Cataract surgery with toric intraocular lens implantation in keratoconus: a case report. Cornea. 2011;30(6):720-3.

11. Luck J. Customized ultra-high-power toric intraocular lens implantation for pellucid marginal degeneration and cataract. J Cataract Refract Surg. 2010;36(7):1235-8.

12. Stewart CM, McAlister JC. Comparison of grafted and non-grafted patients with corneal astigmatism undergoing cataract extraction with a toric intraocular lens implant. Clin Experiment Ophthalmol. 2010;38(8):747-57.

13. Ferrer-Blasco T, Montés-Micó R, Peixoto-de-Matos SC, GonzálezMéijome JM, Cerviño A. Prevalence of corneal astigmatism before cataract surgery. J Cataract Refract Surg. 2009;35(1):70-5. Comment in J Cataract Refract Surg. 2009;35(6):958; author reply 958-9.

Autor correspondente:

Ana Luiza Biancardi

Av. Ataufo de Paiva, $n^{\circ} 245$ - $1^{\circ}$ andar

CEP 22440-032 - Leblon - Rio de Janeiro (RJ), Brasil

Tel: / Fax: 25291350

E-mail: albiancardi@ig.com.br 